\title{
Single-use NPWT device with telemedical wound monitoring in the treatment of acne inversa in a pregnant patient - case report
}

\author{
Kinga Zastawna, Alicja Żalejko-Strychalska, Tomasz Banasiewicz
}

\section{CASE REPORT}

\begin{abstract}
Acne inversa (AI) is a chronic inflammatory skin disease significantly impacting the patient's quality of life. Management guidelines for acne inversa during pregnancy do not exist. We decided to use a single-use negative pressure wound therapy (PICO, Smith \& Nephew) while managing a pregnant patient with AI. Negative pressure wound therapy (NPWT) seemed to improve the patient's condition, managing the exudation, reducing edema, and improving local regeneration, as well as epithelialization. The method was also absolutely safe for the fetus and feasible to conduct in the outpatient department - what was of utmost importance for the patient. The telemedical monitoring of wound healing using mobile technologies seems to be gaining importance for patients and medical practitioners. In the described case, we used the iWound (Polmedi, Poland) application in an outpatient setting.
\end{abstract}

Keywords-acne inversa, NPWT, telemedicine, iWound App, pregnancy, hidradenitis suppurativa

\section{INTRODUCTION}

A CNE inversa (hidradenitis suppurativa) is a chronic inflammatory skin disease, with a significant impact on quality of life! The disorder is generally difficult to treat and in pregnant women! 3 There are limited publications and guidelines delineating treatment of acne inversa during pregnancy. We present a case report of a 30-yearold, pregnant woman, with moderate acne inversa treated with NPWT. We decided to use single-use negative pressure wound therapy (PICO), regarding the patient's pregnancy. The local treatment of inflammation and reduction of septic condition was the main target of therapy. NPWT is as an effective and safe method in this type of wounds. 4 [5

The main aim of this article is to emphasize the importance of vacuum therapy together with the constant telemedical monitoring of the wound healing, what we were able to achieve using iWound App (Polmedi, Poland).

Manuscript received 25.05.2019; revised 20.07.2019. This work did not receive any financial support. TB is the CEO of Polmedi Sp. z o.o.

Author affiliations: Department of General and Endocrine Surgery and Gastroenterological Oncology Department, Poznan University of Medical Sciences, Poznan, Poland, (KZ, AŻS, TB)

*Correspondence to: Kinga Zastawna: kinga.zastawna@gmail.com

\section{PATIENT INFORMATION AND CLINICAL FIDINGS}

We describe the case of a 30 years old female patient, who presented with typical symptoms of acne inversa in the area of the perineum, both groins, and inner tights. At the moment of admission to the hospital, the patient was 16 weeks pregnant. She was diagnosed with acne inversa ten years before the described incident when the symptoms developed in the axillary area, and she underwent surgery with a satisfactory effect. The patient described the present statement as "much worse and unbearable." The symptoms included the presence of painful nodules, abscesses and sinus tracts forming fistulas with a tendency to tissue fibrosis. However, the disease seemed to have no remarkable effect on the pregnancy the patient reported that first symptoms occurred coincidentally with the pregnancy diagnosis. At that stage, she started developing anxiety and symptoms of depression. Due to potential adverse reactions to general anesthesia, we excluded broad surgical excision. We also had limited pharmacotherapy options due to the pregnancy. Additionally, the patient presented no other pathologies and concomitant diseases, no similar cases in the family medical history. The patient did not smoke nor use alcohol.

\section{THERAPEUTIC INTERVENTION}

The patient was admitted to the dermatological ward and received conservative treatment. A team of physicians consisting of a dermatologist, gynecologist, anesthesiologist, and a surgeon were regularly consulting the patient and decided to perform surgical drainage of the inguinal abscesses under the short-term anesthesia. The surgical approach was limited to drainage of the lesions rather than performing the typically recommended radical procedure to avoid a possible compromise to the patient's pregnancy. In patients with AI skin lesions less often cause sepsis or lymphadenopathy, but we were obliged to treat her due to an elevated risk of miscarriage.

The localization of the wounds and lesions was problematic for the regular NPWT dressing as we did not want to limit the patient's movements, so we decided to introduce the NPWT using PICO (Smith \& Nephew) soon after surgery. The small PICO system was easily changeable and removable 

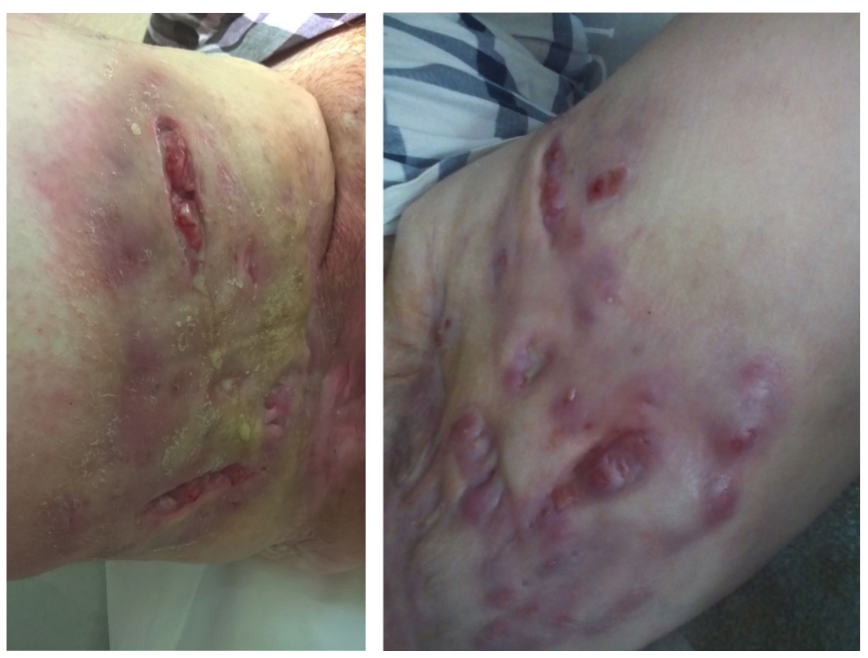

Figure 1. Both treated inguinal areas on the day of the hospital discharge.

by the patient whenever leakage necessary. We continued the NPWT for two weeks after the surgery, with the dressing change every two days in the beginning and every three days later (5 changes in total) and the concomitant intravenous antibiotic therapy. Perioperative antibiotic therapy corresponded to bacteriological tests, performed from lesional skin swabs, taking the potential risk for embryotoxicity in concern (erythromycin $600 \mathrm{mg}$ i.v. three times a day and cefotaxime $2 \mathrm{~g}$ i.v. twice daily for ten days).

After hospitalizing the patient for 22 days, we reached the point when the patient could be discharged from the hospital and instructed about the following home treatment, which included topical application of ichthyol and starch suspension twice daily. To keep the constant monitoring of the patient's wound, we proposed using a smartphone application, what was potentially beneficial due to minimizing the need for outpatient clinic visits. The patient enthusiastically agreed to create an account in the test version of the iWound App (Polmedi, Poland), which offered an easy and fast communication in case of any complications. There was no requirement to keep the patient hospitalized neither in dermatology or surgery department (due to the high risk of infection in both wards) nor in gynecology or obstetrics department (no pregnancy complications during the entire period of pregnancy). We instructed the patient on how to use the iWound App and how often she should contact the doctor. She sent photographs by the application and information about pain and color and amount of discharge every three days right after she left the hospital, and once a week 14 days later. She was also instructed not to hesitate to contact the doctor if any pregnancy-related symptoms would occur. However, she was strongly advised to stay under the obstetrician's care according to his recommendations. After the surgical treatment supported by NPWT, we observed no more severe activity of acne inversa. The patient was monitored as an outpatient until she gave birth in the 38th week. The patient did not require another hospitalization, and the pregnancy was terminated on time by cesarean section.
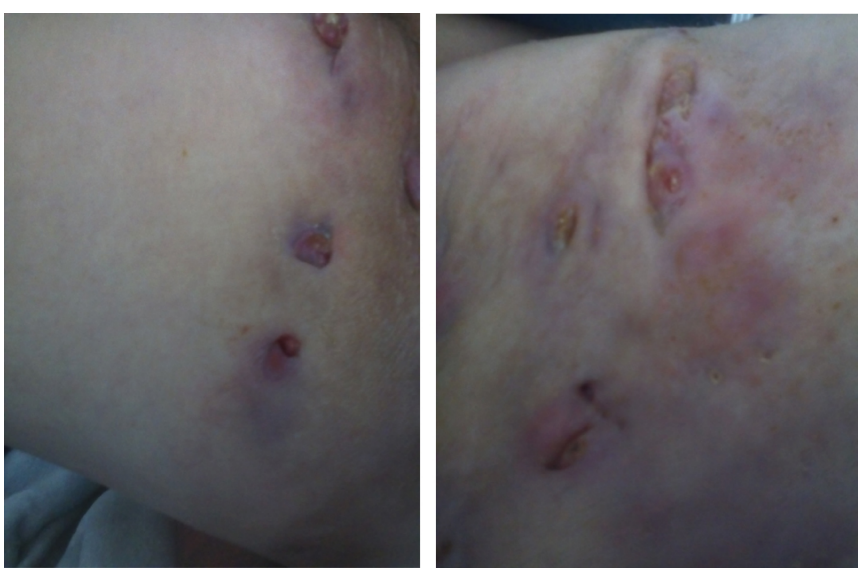

Figure 2. Photographs taken by the patient at home and sent by the iWound App 6 weeks after she was discharged from the hospital.

\section{Discussion}

Although pregnancy is a physiological state, it brings much anxiety when the patient needs hospitalization for unrelated disorders. Being aware of this statement combined with an infectious disease, which unquestionably is acne inversa, we saw the need for a reliable ambulatory monitoring of the patient. The treatment was aimed to minimize the negative impact of AI rather than only introduce radical surgical therapy.

NPWT seemed to be in this case well-suited for improving the healing potential, managing the exudation, reducing edema, improving local regeneration, as well as epithelialization. The method was also, what was crucial for patients, absolutely safe, and feasible to use in the outpatient department. The other benefit was the reduction of the number of wound dressing changes and reducing odor, which posed discomfort for the patient. Single-use NPWT, as in our case, is the easiest way to use NPWT in wound treatment after surgery. The method is simple to use for the patient, and the device operates with just one button. One potential difficulty is the location of the wound dressing, which can introduce problems to keep the vacuum dressing sealed. The experience of the medical staff and the use of stoma paste as a sealant is most suited for the proper functioning of the system, especially in a complicated localization. In our case, we did not observe any significant leaks; no extra visits to the outpatient clinic or emergency wound dressing removal was necessary.

With a holistic view of the patient situation, we tried to address the difficulties that could occur regarding frequent visits, spending time in public transportation or the risk of nosocomial infections in the outpatient clinic. The telemedical application seemed to be well-suited and used by patients and medical staff instruments for monitoring of wound healing with increasing frequency 6 [n the described case the iWound application was used - created by Polmedi, Poland, in collaboration with Chair and Department of General and Endocrine Surgery and Gastroenterological Oncology Department, Poznań University of Medical Sciences, Poznań, Poland. In our and patient's opinion, iWound is very easy to 
use and allows for monitoring and permanent contact with the patient. It is personalized and stores all information together with the photographs of the wound, which is essential due to two aspects. First, it gives the physician the possibility of monitoring patient's healing, second, provides both — the patient and the physician with the visual progress of woundhealing, what is vital from the psychological point of view $[8$

\section{Conclusion}

The ambulatory therapy of the wound, mainly including negative pressure wound therapy, require the regular control of wound healing due to early detection of potential problem and monitoring of the quality of healing. In discharged patients treated with NPWT, it seems to be very important to control the effectiveness of the therapy, early detection of some technical problems (leak, device's alarms), control of type and volume of exudation, as well as to decide when the vacuum dressing should be changed. The smartphone application allows patients to control better and monitor the therapy as well as improve safety. Medical staff can benefit from it by optimizing the frequency of outpatient visits. It can reduce the number of non-necessary visits, as well as allow intervening in case of any acute complications rapidly. In our opinion, ambulatory NPWT treatment can be very effective when combined with telemedical systems for wound healing monitoring; the next step could be direct monitoring of vacuum devices by telemedical monitoring systems or mobile applications.

\section{REFERENCES}

[1] N. Scheinfeld, "Hidradenitis suppurativa: a practical review of possible medical treatments based on over 350 hidradenitis patients," Dermatology online journal, vol. 19, no. 4, 2013.

[2] C. Kromann, I. Deckers, S. Esmann, J. Boer, E. Prens, and G. Jemec, "Risk factors, clinical course and long-term prognosis in hidradenitis suppurativa: a cross-sectional study," British Journal of Dermatology, vol. 171, no. 4, pp. 819-824, 2014.

[3] P. Perng, J. Zampella, and G. Okoye, "Considering the impact of pregnancy on the natural history of hidradenitis suppurativa," British Journal of Dermatology, vol. 178, no. 1, pp. e13-e14, 2018.

[4] E. Chen and H. I. Friedman, "Management of regional hidradenitis suppurativa with vacuum-assisted closure and split thickness skin grafts," Annals of plastic surgery, vol. 67, no. 4, pp. 397-401, 2011.

[5] T. Banasiewicz, A. Bobkiewicz, and M. Borejsza-Wysocki, "Portable vac therapy improve the results of the treatment of the pilonidal sinusrandomized prospective study," Polish Journal of Surgery, vol. 85, no. 7, pp. 371-376, 2013.

[6] P. C. Sanger, A. Hartzler, S. M. Han, C. A. Armstrong, M. R. Stewart, R. J. Lordon, W. B. Lober, and H. L. Evans, "Patient perspectives on post-discharge surgical site infections: towards a patient-centered mobile health solution," PLoS One, vol. 9, no. 12, p. e114016, 2014.

[7] J. S. Abelson, M. Symer, A. Peters, M. Charlson, and H. Yeo, "Mobile health apps and recovery after surgery: What are patients willing to do?" The American Journal of Surgery, vol. 214, no. 4, pp. 616-622, 2017.

[8] J. Ye, Y. Zuo, T. Xie, M. Wu, P. Ni, Y. Kang, X. Yu, X. Sun, Y. Huang, and S. Lu, "A telemedicine wound care model using $4 \mathrm{~g}$ with smart phones or smart glasses: A pilot study," Medicine, vol. 95, no. 31, 2016. 